\title{
ARMAZENAMENTO DE MAÇÃS 'ROYAL GALA' SOB DIFERENTES TEMPERATURAS E PRESSÕES PARCIAIS DE OXIGÊNIO E GÁS CARBÔNICO ${ }^{1}$
}

\author{
AURI BRACKMANN ${ }^{2}$, ANDERSON MACHADO DE MELLO ${ }^{3}$, \\ SÉRGIO TONETTO DE FREITAS ${ }^{3}$, MÁRCIA VIZZOTTO ${ }^{4}$, CRISTIANO ANDRÉ STÉFFENS ${ }^{4}$
}

\begin{abstract}
RESUMO - O experimento foi conduzido com o objetivo de avaliar o efeito da temperatura de armazenamento e condições de atmosfera controlada sobre a manutenção da qualidade da maçã cv. Royal Gala. O delineamento experimental utilizado foi o inteiramente casualizado, arranjado em um esquema bifatorial, com 4 repetições e a amostra composta por 25 frutos. Os oito tratamentos originaram-se da combinação de dois níveis do fator temperatura $\left(-0.5^{\circ} \mathrm{Ce} 0^{\circ} \mathrm{C}\right)$ com quatro níveis do fator condição de atmosfera controlada $\left(1 \mathrm{kPa} \mathrm{O}_{2} /\right.$ $2 \mathrm{kPa} \mathrm{CO}_{2}, 1,2 \mathrm{kPa} \mathrm{O}_{2} / 2 \mathrm{kPa} \mathrm{CO}_{2}, 1 \mathrm{kPa}$ de $\mathrm{O}_{2} / 3 \mathrm{kPa} \mathrm{CO} 2$ e $1,2 \mathrm{kPa} \mathrm{O}_{2} / 3 \mathrm{kPa} \mathrm{CO}_{2}$ ). As avaliações foram realizadas após nove meses de armazenamento, na abertura das câmaras, e após sete dias de exposição dos frutos à temperatura de $20^{\circ} \mathrm{C}$. De acordo com os resultados obtidos, as temperaturas testadas não diferiram estatisticamente com relação à acidez titulável na saída da câmara, sendo que a melhor combinação de gases foi $1,2 \mathrm{kPa}$ de $\mathrm{O}_{2}$ e $2 \mathrm{kPa}$ de $\mathrm{CO}_{2}$. Já após sete dias de armazenamento a $0^{\circ} \mathrm{C}$, não houve diferença estatística com relação à concentração de gases. A temperatura de $0^{\circ} \mathrm{C}$ apresentou maior firmeza de polpa, menor incidência de frutos com polpa farinhenta e degenerescência da polpa após sete dias a $20^{\circ} \mathrm{C}$. A combinação de gases $1,0 \mathrm{kPa}$ de $\mathrm{O}_{2}$ e 2 e $3 \mathrm{kPa}$ de $\mathrm{CO}_{2}$ apresentou melhor manutenção da acidez titulável e menores valores de ocorrência de podridões e distúrbios fisiológicos. Concluiu-se que a melhor temperatura de armazenamento para a cv. Royal Gala é de $0^{\circ} \mathrm{C}$ e a melhor combinação de gases é de $1 \mathrm{kPa}$ de $\mathrm{O}_{2}$ e 2 e $3 \mathrm{kPa}$ de $\mathrm{CO}_{2}$.
\end{abstract}

Termos para indexação: atmosfera controlada, qualidade, distúrbio fisiológico, Malus domestica.

\section{STORAGE OF 'ROYAL GALA' APPLE UNDER DIFFERENT TEMPERATURES AND CARBON DIOXIDE AND OXYGEN PARTIAL PRESSURE}

\begin{abstract}
This work was conducted to evaluate the effect of temperature and CA storage conditions on the quality of 'Royal Gala' apples. The experiemtal desig was completely randomized with 4 replications of 25 fruits. Evaluated temperatures were $-0,5^{\circ} \mathrm{C}$ and $0{ }^{\circ} \mathrm{C}$ combined with following $\mathrm{O}_{2}$ and $\mathrm{CO}_{2}$ partial pressures: $1 \mathrm{kPa} \mathrm{O} / 2 \mathrm{kPa} \mathrm{CO}_{2}, 1,2 \mathrm{kPa} \mathrm{O}_{2} / 2 \mathrm{kPa} \mathrm{CO}_{2}, 1 \mathrm{kPaO}_{2} / 3 \mathrm{kPa} \mathrm{CO}_{2}$ and $1,2 \mathrm{kPa} \mathrm{O} / 3 \mathrm{kPa}$ $\mathrm{CO}_{2}$. Fruit quality was assessed after 9 months of storage, at the openning of the chanbers and after 7 days of shelf life at $20^{\circ} \mathrm{C}$. Results showed that $0^{\circ} \mathrm{C}$ fruit had higher titratable acidity at the openning of the chambers and higher pulp fiirmness, lower mealness and internal breakdown incidence after 7 days of shelf life. The best CA conditions was $1,2 \mathrm{kPa} \mathrm{O}_{2}$ with 2 and $3 \mathrm{kPa} \mathrm{CO}_{2}$ wich presented highest titratable acidity and less incidence of rot and physiological disorders.
\end{abstract}

Index terms : controlled atmosphere, quality, physiological disorder, Malus domestica..

\section{INTRODUÇÃO}

A temperatura de armazenamento exerce importante papel na conservação das qualidades físico-químicas dos frutos e na prevenção e, ou diminuição da incidência de doenças e distúrbios fisiológicos. $\mathrm{O}$ abaixamento da temperatura diminui a respiração dos frutos (Childers, 1975) e retarda o amadurecimento (Bartley, 1974), resultando em maior período de conservação (Lyons, 1975).

A cv. Gala comporta-se muito bem em temperaturas entre $0^{\circ} \mathrm{C}$ e $2^{\circ} \mathrm{C}$, não apresentando perdas acentuadas na firmeza de polpa (Little \& Peggie, 1987). Bender (1989b) observou que temperaturas de $0 \mathrm{a} 1^{\circ} \mathrm{C}$ conservam maçãs por um período máximo de cinco meses. Entretanto, caso a temperatura permaneça muito baixa, pode ocasionar ocorrência de desordens fisiológicas (Fidler \& North, 1967).
Inúmeros trabalhos têm demonstrado os efeitos da atmosfera controlada (AC) sobre a fisiologia e qualidade de maçãs durante $o$ armazenamento. Os efeitos benéficos do armazenamento de maçãs sob baixas pressões parciais de $\mathrm{O}_{2} \mathrm{e}$ altas pressões parciais de $\mathrm{CO}_{2}$ incluem redução da taxa respiratória e de produção de etileno, conservando, assim, as características físico-química e inibindo a incidência de alguns distúrbios fisiológicos. A cv. Gala, por exemplo, pode ser armazenada em atmosfera controlada por até 8 meses (Hansen \& Zanon, 1982).

As pressões parciais de $\mathrm{O}_{2}$ e $\mathrm{CO}_{2}$ adequadas para o armazenamento de maçã 'Gala' diferem em função do local e ano de colheita (Sharples \& Johnson, 1987; Meheriuk, 1989). A redução da pressão parcial de $\mathrm{O}_{2}$ é necessária para reduzir a respiração, porém, deve-se observar um valor mínimo entre $1 \mathrm{kPa}$

\footnotetext{
1 (Trabalho 249/2000). Recebido: 13/11/2000. Aceito para publicação: 04/10/2001.

2 Engenheiro Agrônomo, Doutor, Professor do Depto. de Fitotecnia, Centro de Ciências Rurais, Universidade Federal de Santa Maria, $97105-$ 900, Santa Maria-RS.

3 Acadêmicos do Curso de Agronomia, Universidade Federal de Santa Maria, Santa Maria-RS. Bolsista/CNPq.

4 Engenheiros Agrônomos, alunos do PPGA em Agronomia da Universidade Federal de Santa Maria.
} 
e $3 \mathrm{kPa}$, dependendo do produto e tempo de exposição. Isso é para evitar o processo de respiração anaeróbica. $\mathrm{O} \mathrm{CO}_{2}$, quando em níveis elevados, também diminui a taxa respiratória dos frutos, mas, quando em pressão parcial muito alta, pode causar danos (Kader, 1985). Meheriuk (1993) recomenda para o armazenamento da cv. Gala, nas condições de cultivo do Brasil, pressões parciais de $1,5 \mathrm{kPa}$ a $2 \mathrm{kPa}$ de $\mathrm{O}_{2}$ e $2,5 \mathrm{kPa}$ a $3 \mathrm{kPa}$ de $\mathrm{CO}_{2}$, apresentando melhores resultados de qualidade de frutos na avaliação realizada na saída da câmara. Já após cinco dias de exposição à temperatura ambiente, os melhores resultados foram obtidos com $1 \mathrm{kPa}$ de $\mathrm{O}_{2}$ e 1 a $3 \mathrm{kPa}$ de $\mathrm{CO}_{2}$, salientando que essa cultivar é sensível a altas pressões parciais de gás carbônico, podendo causar distúrbios fisiológicos.

O objetivo desse trabalho foi determinar o efeito de temperaturas de armazenamento e diferentes condições de AC sobre as qualidades físico-químicas e ocorrência de distúrbios fisiológicos e podridões em maçãs cv. Royal Gala.

\section{MATERIAL E MÉTODOS}

O experimento de armazenamento de maçã cv. Royal Gala foi conduzido no Núcleo de Pesquisa em Pós-colheita (NPP) do Departamento de Fitotecnia da Universidade Federal de Santa Maria-RS. Os frutos foram colhidos em pomar comercial pertencente à empresa Agropecuária Schio de Vacaria (RS), com ponto de maturação utilizado pela empresa, ou seja, com índice iodo-amido igual a 5,46. Ainda na empresa, os frutos foram selecionados e classificados, utilizando-se do calibre 135, que equivale ao número de frutos por caixa de $18 \mathrm{~kg}$. Após o transporte dos frutos à UFSM, em Santa Maria, procedeu-se nova seleção destes, eliminando aqueles com lesões e/ou defeitos e, posteriormente, homogeneizaram-se as amostras experimentais.

Para o armazenamento, foram utilizadas minicâmaras experimentais de $\mathrm{AC}$, com volume de 232 litros, as quais foram conectadas por tubulações plásticas a um equipamento de controle automático das pressões parciais de gases da marca Kronenberger Sistemtechnik. As minicâmaras permaneceram no interior de câmaras frigoríficas de $45 \mathrm{~m}^{3}$, com sistema de refrigeração por ar forçado, nas temperaturas de $-0,5^{\circ} \mathrm{C} \mathrm{e} 0^{\circ} \mathrm{C}$.

Após a colocação das amostras dentro das minicâmaras, foram instaladas as atmosferas, conforme as pressões parciais de $\mathrm{O}_{2}$ e $\mathrm{CO}_{2}$ estabelecidas nos tratamentos. As pressões parciais iniciais de $\mathrm{O}_{2}$ foram obtidas pelo princípio da diluição do $\mathrm{O}_{2}$, com a injeção de gás nitrogênio, proveniente de um gerador de nitrogênio que utiliza o princípio "Pressure Swing Adsorption" (PSA), até a obtenção das concentrações parciais preestabelecidas. As concentrações parciais de $\mathrm{CO}_{2}$ foram obtidas através da injeção deste gás nas minicâmaras.

Para a manutenção constante dos níveis de $\mathrm{O}_{2}$ e $\mathrm{CO}_{2}$, que continuamente se modificavam em função do processo respiratório dos frutos, foram realizadas diariamente três análises e correções das pressões parciais dos gases, e pelo sistema automático de controle dos gases. $\mathrm{O} \mathrm{O}_{2}$ consumido pela respiração foi reposto através da injeção de ar atmosférico nas minicâmaras. $\mathrm{O} \mathrm{CO}_{2}$ em excesso foi absorvido pelo absorvedor de $\mathrm{CO}_{2}$ que utiliza uma solução de hidróxido de potássio (40\%).

A temperatura foi controlada automaticamente por termostatos de alta precisão, sendo monitorada diariamente, utilizando termômetros de mercúrio introduzidos na polpa de frutos que apresentaram uma oscilação de $\pm 0,2^{\circ} \mathrm{C}$.

Após nove meses de armazenamento, os frutos foram retirados das minicâmaras e separados em oito amostras de 25 frutos. Quatro amostras foram analisadas no mesmo dia e as outras quatro, após uma semana de exposição à temperatura de $20^{\circ} \mathrm{C}$, em câmara de climatização. Para cada tratamento, foram calculadas a média e a variância das variáveis avaliadas, sendo as médias comparadas pelo teste de Duncan (5\%).

As variáveis teste iodo-amido, firmeza da polpa, sólidos solúveis totais (SST), acidez titulável, podridões e degenerescência da polpa dos frutos e polpa farinhenta foram avaliadas conforme metodologia citada por Brackmann \& Saquet (1995).

\section{RESULTADOS E DISCUSSÃO}

Após nove meses de armazenamento, a firmeza de polpa, na saída da câmara, apresentou-se menor nos frutos armazenados em $1,2 \mathrm{kPa}$ de $\mathrm{O}_{2}$ e $2 \mathrm{kPa}$ de $\mathrm{CO}_{2}$, na temperatura de $-0,5^{\circ} \mathrm{C}$ (Tabela 1). Após sete dias a $20^{\circ} \mathrm{C}$, a melhor combinação de gases para a manutenção da firmeza de polpa foi de $1 \mathrm{kPa} \mathrm{de}_{2}$ e $3 \mathrm{kPa}$ de $\mathrm{CO}_{2}$ (Tabela $1 \mathrm{e} 3$ ). Esta combinação de gases está bastante próxima à obtida por outros autores que demonstram que o uso de altas pressões de $\mathrm{CO}_{2}$ mantém maior a firmeza de polpa durante o armazenamento (Anderson, 1967; North \& Cockburn, 1978). Esses autores também afirmam que o aumento de $\mathrm{CO}_{2}$ apresenta maior efeito sobre a redução dos processos metabólicos dos frutos que uma leve redução do $\mathrm{O}_{2}$. A temperatura de armazenamento que manteve maior a firmeza de polpa foi de $0^{\circ} \mathrm{C}$, concordando com Bender (1989) que recomenda temperaturas de $0^{\circ} \mathrm{C}$ a $1^{\circ} \mathrm{C}$ para a cv. Gala.

A acidez titulável apresentou, na saída da câmara, valores que não diferiram estatisticamente em relação à temperatura de armazenamento, sendo que a melhor combinação de gases foi de $1,2 \mathrm{kPa}$ de $\mathrm{O}_{2}$ e $2 \mathrm{kPa}$ de $\mathrm{CO}_{2}$ (Tabela 1). Já após sete dias, não houve diferença estatística entre as condições de AC ( Tabela 3). $\mathrm{Na}$ temperatura de $0^{\circ} \mathrm{C}$, os frutos armazenados em $1 \mathrm{kPa}$ de $\mathrm{O}_{2}$ com $2 \mathrm{kPa}$ de $\mathrm{CO}_{2}$ e $1,2 \mathrm{kPa}$ de $\mathrm{O}_{2}$ com $3 \mathrm{kPa}$ de $\mathrm{CO}_{2}$ apresentaram os maiores níveis de acidez titulável, podendo isto ser atribuído à menor taxa respiratória dos frutos. Alguns autores, como Lidster et al. (1980), com a cv. McIntosh, e Brackmann \& Saquet (1995), com a cv. Gala, afirmam que pressões parciais de $\mathrm{O}_{2}$ próximo a $1 \mathrm{kPa}$ ou menos mantém a acidez dos frutos mais elevada.

$\mathrm{Na}$ avaliação do teor de sólidos solúveis totais (SST), tanto na saída da câmara quanto após sete dias(Tabelas 1 e 3), os tratamentos não diferiram estatisticamente com relação às condições de AC, confirmando o resultado obtido por Brackmann $\&$ Saquet (1995). Provavelmente, isto é devido ao fato de que os SSTs são degradados pela respiração após uma acentuada degradação dos ácidos orgânicos e, além disso, a degradação é mais lenta em AC (atmosfera controlada) do que em atmosfera refrigerada normal (Brackmann, 1990). Estes resultados estão de acordo com os obtidos por Lidster et al. (1980), que também não verificaram diferenças significativas nos SSTs em decorrência do baixo $\mathrm{O}_{2}(1$ e $1,5 \mathrm{kPa})$. Porém, houve diferença significativa com relação à temperatura de armazenamento, sendo que a $0^{\circ} \mathrm{C}$ 
TABELA 1 - Qualidades físico-químicas de maçãs cv. Royal Gala após nove meses de armazenamento, em condições de AC, em duas temperaturas. Santa Maria-RS, 2000.

\begin{tabular}{|c|c|c|c|c|c|c|c|c|}
\hline \multirow[t]{2}{*}{$\begin{array}{c}\mathrm{O} 2 / \mathrm{COO} 2 \\
(\mathrm{kPa})\end{array}$} & \multicolumn{2}{|c|}{$\begin{array}{c}\text { Firmeza de polpa } \\
(N)\end{array}$} & \multicolumn{3}{|c|}{$\begin{array}{c}\text { A cidez titulável } \\
(\text { (cmol/L) }\end{array}$} & \multicolumn{3}{|c|}{$\begin{array}{c}\text { SS T } \\
\left({ }^{\circ} \text { B rix }\right)\end{array}$} \\
\hline & $\overline{0^{\circ} \mathrm{C}}$ & $-0,5^{\circ} \mathrm{C}$ & $0^{\circ} \mathrm{C}$ & $-0,5^{\circ} \mathrm{C}$ & M édia & $0^{\circ} \mathrm{C}$ & $-0,5^{\circ} \mathrm{C}$ & Média \\
\hline $1 / 2$ & $78,08 \mathrm{Aa}^{*}$ & $84,27 \mathrm{~A} \mathrm{a}$ & 4,65 & 4,58 & $4,61 \mathrm{~b}$ & 13,6 & 13,3 & $13,4 \mathrm{a}$ \\
\hline $1,2 / 2$ & $84,27 \mathrm{~A} \mathrm{a}$ & $72,68 \mathrm{~B} \mathrm{~b}$ & 4,81 & 4,81 & $4,81 \mathrm{a}$ & 13,9 & 13,4 & $13,6 \mathrm{a}$ \\
\hline $1 / 3$ & $81,13 \mathrm{~A}$ a & $77,96 \mathrm{~A} \mathrm{ab}$ & 4,44 & 4,56 & $4,50 \mathrm{~b}$ & 13,6 & 13,2 & $13,4^{\mathrm{a}}$ \\
\hline $1,2 / 3$ & $82,95 \mathrm{~A}$ a & $78,94 \mathrm{~A} \mathrm{ab}$ & 4,45 & 4,62 & $4,56 \mathrm{~b}$ & 13,6 & 13,5 & $13,5 \mathrm{a}$ \\
\hline M édia & & & $4,6 \mathrm{~A}$ & $4,6 \mathrm{~A}$ & & $13,7 \mathrm{f}$ & $13,4 \mathrm{~B}$ & \\
\hline
\end{tabular}

*Médias seguidas de mesma letra não diferem estatisticamente, pelo teste de Duncan (5\%).

TABELA 2 - Ocorrência de distúrbios fisiológicos e podridões em maçãs cv. Royal Gala após nove meses de armazenamento, em condições de AC, em duas temperaturas. Santa Maria- RS,2000

\begin{tabular}{|c|c|c|c|c|c|c|c|c|c|}
\hline \multirow[t]{2}{*}{$\begin{array}{c}\mathrm{O2} / \mathrm{CO2} \\
(\mathrm{kPa})\end{array}$} & \multicolumn{3}{|c|}{$\begin{array}{c}\text { Polpa farinhenta } \\
(\%)\end{array}$} & \multicolumn{3}{|c|}{$\begin{array}{c}\text { Degenerescência } \\
\text { Senescente }(\%)\end{array}$} & \multicolumn{3}{|c|}{$\begin{array}{c}\text { Podridão } \\
(\%)\end{array}$} \\
\hline & $0^{\circ} \mathrm{C}$ & $-0,5^{\circ} \mathrm{C}$ & M édia & $0^{\circ} \mathrm{C}$ & $-0,5^{\circ} \mathrm{C}$ & M édia & $0^{\circ} \mathrm{C}$ & $-0,5^{\circ} \mathrm{C}$ & M édia \\
\hline $1,2 / 2$ & 8,32 & 3,75 & $6,01 \mathrm{a}$ & 3,89 & 5,00 & $4,4 \mathrm{ab}$ & 2,5 & 1,25 & $1,9 \mathrm{a}$ \\
\hline M édia & $6,5 \mathrm{~A}$ & $4,7 \mathrm{~A}$ & & $4,3 \mathrm{~A}$ & $5,0 \mathrm{~A}$ & & $0,6 \mathrm{~A}$ & $0,6 \mathrm{~A}$ & \\
\hline
\end{tabular}

* Médias seguidas de mesma letra não diferem estatisticamente, pelo teste de Duncan (5\%).

TABELA 3 - Qualidades físico-químicas de maçãs cv. Royal Gala após nove meses de armazenamento em AC e sete dias de exposição à temperatura de $20^{\circ} \mathrm{C}$. Santa Maria-RS,2000.

\begin{tabular}{|c|c|c|c|c|c|c|c|c|}
\hline \multirow[t]{2}{*}{$\begin{array}{c}\mathrm{O2} / \mathrm{CO2} \\
(\mathrm{kPa})\end{array}$} & \multicolumn{3}{|c|}{$\begin{array}{c}\text { Firmeza de polpa } \\
\text { (N) }\end{array}$} & \multicolumn{2}{|c|}{$\begin{array}{l}\text { Acidez titulável } \\
\text { (cmol/L) }\end{array}$} & \multicolumn{3}{|c|}{$\begin{array}{c}\text { SST } \\
\left({ }^{\circ} \text { B rix }\right)\end{array}$} \\
\hline & $0^{\circ} \mathrm{C}$ & $-0,5^{\circ} \mathrm{C}$ & Média & $0^{\circ} \mathrm{C}$ & $-0,5^{\circ} \mathrm{C}$ & $0^{\circ} \mathrm{C}$ & $-0,5^{\circ} \mathrm{C}$ & Média \\
\hline $1 / 2$ & 19,67 & $\overline{17,50}$ & $82,69 b^{*}$ & $4,65 \mathrm{Aa}$ & $\overline{4,26 \mathrm{Ba}}$ & 13,65 & 13,35 & $\overline{13,50 \mathrm{a}}$ \\
\hline $1,2 / 2$ & 19,85 & 18,24 & $84,64 \mathrm{ab}$ & $4,51 \mathrm{Aab}$ & $4,34 \mathrm{Aa}$ & 13,45 & 13,32 & $13,40 \mathrm{a}$ \\
\hline $1 / 3$ & 20,44 & 18,76 & $87,20 \mathrm{a}$ & $4,36 \mathrm{Ab}$ & $4,39 \mathrm{Aa}$ & 13,40 & 13,27 & $13,20 \mathrm{a}$ \\
\hline $1,2 / 3$ & 19,21 & 18,15 & $83,10 \mathrm{~b}$ & $4,65 \mathrm{Aa}$ & $4,29 \mathrm{Ba}$ & 13,50 & 13,50 & $13,50 \mathrm{a}$ \\
\hline Média & $19,79 \mathrm{~A}$ & $18,16 \mathrm{~B}$ & & & & $13,50 \mathrm{~A}$ & $13,30 \mathrm{~B}$ & \\
\hline
\end{tabular}

*Médias seguidas de mesma letra não diferem estatisticamente, pelo teste de Duncan (5\%).

TABELA 4 - Ocorrência de distúrbios físiológicos e podridões em maçãs cv. Royal Gala após nove meses de armazenamento em AC e sete dias de exposição à temperatura de $20^{\circ} \mathrm{C}$.Santa Maria-RS, 2000.

\begin{tabular}{|c|c|c|c|c|c|c|c|c|}
\hline \multirow[t]{2}{*}{ 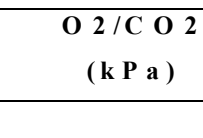 } & \multicolumn{3}{|c|}{$\begin{array}{c}\text { Polpa farin henta } \\
(\%)\end{array}$} & \multicolumn{2}{|c|}{$\begin{array}{c}\text { De generescên cia } \\
\text { Senescente (\%) }\end{array}$} & \multicolumn{3}{|c|}{$\begin{array}{c}\text { Podrid ão } \\
(\%)\end{array}$} \\
\hline & $0^{\circ} \mathrm{C}$ & $-0,5^{\circ} \mathrm{C}$ & M éd ia & $0^{\circ} \mathrm{C}$ & $-0,5^{\circ} \mathrm{C}$ & $0^{\circ} \mathrm{C}$ & $-0,5^{\circ} \mathrm{C}$ & Média \\
\hline $1 / 2$ & 5,0 & 11,25 & $8,1 \mathrm{a} *$ & $8,8 \mathrm{~B} \mathrm{a}$ & $37,5 \mathrm{~A} \mathrm{a}$ & 2,5 & 2,5 & $2,5 \mathrm{a}$ \\
\hline $1,2 / 2$ & 2,5 & 8,75 & $5,6 \mathrm{a}$ & $7,8 \mathrm{~B} \mathrm{a}$ & $17,5 \mathrm{~A} \mathrm{~b} \mathrm{c}$ & 5,0 & 1,25 & $3,1 \mathrm{a}$ \\
\hline $1 / 3$ & 0,0 & 6,25 & $3,1 \mathrm{a}$ & $3,8 \mathrm{~B} \mathrm{a}$ & $30,0 \mathrm{~A} \mathrm{ab}$ & 2,5 & 2,5 & $2,5 \mathrm{a}$ \\
\hline $1,2 / 3$ & 6,25 & 6,07 & $6,2 \mathrm{a}$ & $10,0 \mathrm{~A}$ a & $13,6 \mathrm{Ac}$ & 2,5 & 4,76 & $3,6 \mathrm{a}$ \\
\hline
\end{tabular}

* Médias seguidas de mesma letra não diferem estatisticamente, pelo teste de Duncan (5\%). 
manteve-se um valor mais elevado de SST. Não houve diferença estatística em relação à incidência de frutos com polpa farinácea em função das pressões parciais de $\mathrm{O}_{2}$ e $\mathrm{CO}_{2}$, tanto na saída da câmara quanto após sete dias de exposição à temperatura de $20^{\circ} \mathrm{C}$ (Tabelas 2 e 4). Quanto à temperatura de armazenamento, após sete dias a $20^{\circ} \mathrm{C}$, a temperatura de $0,5^{\circ} \mathrm{C}$ apresentou maior incidência de frutos com polpa farinácea. Isso pode ser explicado pelo fato de os tratamentos a $-0,5^{\circ} \mathrm{C}$ apresentarem uma menor média da firmeza de polpa na saída da câmara e uma porcentagem maior da degenerescência senescente após sete dias a $20^{\circ} \mathrm{C}$.

Não houve diferença estatística em relação à ocorrência de degenerescência senescente, na saída da câmara, em função da variação da temperatura e da pressão parcial dos gases durante o armazenamento ( Tabela 2). Já após sete dias de exposição ao ambiente, a temperatura de armazenamento que apresentou uma maior incidência de frutos com degenerescência senescente foi a de $-0,5^{\circ} \mathrm{C}$ (Tabela 4). Segundo Lyons (1975), a temperatura de armazenamento, quando muito baixa, influi nos complexos enzimáticos e permeabilidade das membranas, solidificando a parte lipídica. Fortes \& Petri (1982) afirmam que a suscetibilidade de maçãs ao $\mathrm{CO}_{2}$ aumenta com a redução de temperatura, e de acordo com Little \& Peggie (1987), a cv. Gala pode apresentar danos por baixas temperaturas. De acordo com Ebert (1984), este dano pode ocorrer na cv. Gala após longos períodos de armazenamento, sendo que a mudança bioquímica mais importante no tecido afetado é a acumulação de acetoaldeído, que pode também induzir a degenerescência da polpa dos frutos.

Não houve diferença estatística no número de frutos podres, em relação à temperatura de armazenamento, tanto na saída da câmara como após sete dias de exposição dos frutos à temperatura de $20^{\circ} \mathrm{C}$ (Tabelas 2 e 4 ). A combinação de gases que apresentou maior incidência de frutos podres foi $1,2 \mathrm{kPa}$ de $\mathrm{O}_{2} /$ $2 \mathrm{kPa}$ de $\mathrm{CO}_{2}$. Brackmann \& Saquet (1995) também verificaram maior incidência de frutos podres nos tratamentos com $\mathrm{CO}_{2}$ mais baixo e $\mathrm{O}_{2}$ mais elevado. Entretanto, após sete dias a $20^{\circ} \mathrm{C}$, não houve diferença estatística entre as condições de AC.

Pelos resultados obtidos, podemos observar que a melhor temperatura de armazenamento é de $0^{\circ} \mathrm{C}$, pois proporciona frutos com maior acidez titulável, na saída da câmara, e maior firmeza de polpa, menor incidência de frutos com polpa farinácea e degenerescência, após sete dias a $20^{\circ} \mathrm{C}$. Em relação à atmosfera controlada, foi verificado que a melhor combinação de gases é de $1 \mathrm{kPa}$ de $\mathrm{O}_{2}$ com 2 e $3 \mathrm{kPa}$ de $\mathrm{CO}_{2}$, pois os frutos armazenados nestas condições apresentaram satisfatória manutenção da acidez titulável e menores valores de ocorrência de podridões e distúrbios fisiológicos.

\section{CONCLUSÃO}

A melhor temperatura para o armazenamento de maçãs cultivar Royal Gala é $0^{\circ} \mathrm{C}$ e $1 \mathrm{kPa}$ de $\mathrm{O}_{2} \operatorname{com} 2$ ou $3 \mathrm{kPa}$ de $\mathrm{CO}_{2}$ a melhor combinação de gases.

\section{REFERÊNCIAS BIBLIOGRÁFICAS}

ANDERSON, R.E. Experimental storage of Eastern-grown 'Delicious' apples in various controlled atmospheres.
Proceedings of the American Society of Horticultural Science, East Lansing, v.91, p. 810-820,1967.

BARTLEY, I.M. â-galactosidase activity in ripening apples. Phytochemistry, Oxford, v.13, n.10, p.2107-2111, 1974.

BENDER,R. J. Frigoconservação convencional e em atmosfera controlada de maçãs cv. Gala. Revista Brasileira de Fruticultura, Cruz das Almas, v. 11, n. 1, p. 45-50, 1989a

BENDER, R J.Controlled atmosphere storage of apples cv. Gala in southern Brazil. Acta Horticulturae, Wageningen, n. 258, p.221$223,1989 b$.

BENDER, R.J. Frigoconservação de maçã: a atmosfera controlada. Agropecuária Catarinense, Florianópolis, v.2, n.1, p.56-57, 1989.

BRACKMANN, A. Einflub von Lagerung unter kontrollierter Atmosphäre (CA) und Ethylenbehandlungen auf verschiedene Merkmale der Fruchtreife unter desonderer Berücksichtigung der Aromabildung bei Äpfeln. 1990. 115p. Dissertation (Doktors der Agrarwissenschaften) - Institut für Obst - Gemüse und Weinbau, Universität Hohenheim, 1990.

BRACKMANN, A.; SAQUET, A. A. Armazenamento de maçã cv. Gala em atmosfera controlada. Revista Brasileira de Agrociência., Pelotas, v.1, n.2, p. 55-60, 1995.

CHILDERS, N. F. Modern fruit science. 6. ed. Somerville: Somerset Press, 1975. 1976p.

EBERT, A. Distúrbios fisiológicos. In: CURSO SOBRE A CULTURA DA MACIEIRA, 2., 1984, Caçador: EMPASC. Palstras... p.129-134.

FIDLER, J.; NORTH, C. J. The effect of conditions of storage on the respiration of apples. I. The effects of temperature and concentrations of carbon dioxide and oxygen on the production of carbon dioxide and uptake of oxigen. Journal of Horticultural Science, London, v.42, p.189-206, 1967.

FORTES, G. R. L.; PETRI, J. L. Distúrbios fisológicos em macieira e seu controle. Florianópolis: EMPASC/ACARESC,1989. 34p. (Boletim Técnico, 3)

HANSEN, H.; ZANON, K. Apfelsorte 'Gala' und ihre Mutante 'Royal Gala'. Erwerbsobstbau, Berlin, n.4, p.105-108, 1982.

KADER, A. A. Na overview of the physiological and biochemical basic of CA effects on fresh horticultural crops. In: BLANKENSHIP, S. M. (Ed.) Controlled atmospheres for storange and transport of perishable agricultural comodities. Raleigh, 1985. p. 1-9.

LINDTER, P. D.;FORSYTH, F.R.;LIGHTFOOT, H.J. low oxygen and carbon dioxide atmospheres for storage of 'MeIntosh' apples. Canadian of Journal Plant Science, Ottawa, v.60, p.299-301, 1980.

LITTLE,C.R.;PEGGIE,I.D. Storage injury of pome fruit caused by 
stress levels of oxygen, carbon dioxide, temperature and ethylene. HortScience, Alexandria, v.22, n.5, p.783-790, 1987.

LYONS, J. M. The influence of temperature on fruit ripening. Colloques Internationaux, C.N.R.S., Paris, n.238, p.17-21,1975.

MEHERIUK, M. CA storage conditions for apples, pears and nashe. In: CA 93 FROM CONTR. ATM. RES. CONF. CA '93, 1993, New York. Proceedings... v.2, p.819-858.
NORTH, C. J.; COCKBURN, J.T. Effects of increased concentrations of $\mathrm{CO}_{2}$ on the storage of Cox's Orange Pippin apples in $1 \% \mathrm{O}_{2}$. Report e. Malling. Res. Stn.For 1977, Maidstone, p.146-147, 1978.

SHARPLES, R. O. ; JOHNSON, D. S. Influence of agronomic and climatic factrors on the response of apple fruit to controlled atmosphere storange. Hortscience, Alexandria, v.22, n..5, p.763766, 1987. 